\title{
Targeted sequencing of cancer-associated genes in hepatocellular carcinoma using next generation sequencing
}

\author{
JIANGUO LU ${ }^{1 *}$, JIKAI YIN $^{1 *}$, RUI DONG ${ }^{1}$, TAO YANG $^{1}$, LIJUAN YUAN $^{1}$, \\ $\mathrm{LI} \mathrm{ZANG}^{1}$, CHENG XU ${ }^{2}$, BO PENG ${ }^{2}$, JIANGMAN ZHAO ${ }^{2}$ and XILIN DU ${ }^{1}$ \\ ${ }^{1}$ Department of General Surgery, Tangdu Hospital, Fourth Military Medical University, Xi'an, Shaanxi 710038; \\ ${ }^{2}$ Department of Medicine, Shanghai Zhangjiang Translational Medicine Research Center, Shanghai 201203, P.R. China
}

Received August 4, 2014; Accepted April 20, 2015

DOI: $10.3892 / \mathrm{mmr} .2015 .3952$

\begin{abstract}
Liver cancer is one of the most common causes of cancer-associated mortality. Hepatocellular carcinoma (HCC) is the major histological subtype among types of primary liver cancer. China is an area of high incidence of $\mathrm{HCC}$, and $>50 \%$ of the cases of HCC worldwide are in China. At present, the mechanism underlying the development of HCC remains to be fully elucidated, and previous studies have predominantly focused on HCC in southern and eastern China, with molecular data of the HCC cases in Western China remains limited. In the present study, a panel of 372 cancer-associated genes were screened using a next generation sequencing platform, which included a total of 12 cases from western China. The results confirmed mutations in previously identified HCC drivers, including p53 and Kras. Additionally, mutations in several cancer genes, which had not been previously associated with HCC, were identified, including RUNX1 and JAK3. The present study provided a mutation spectrum of HCC tissue in cases from western China, assisting in the investigation of the mechanism of liver carcinogenesis.
\end{abstract}

\section{Introduction}

Liver cancer is one of the most common types of cancer and is the third most frequent cause of cancer-associated mortality. Hepatocellular carcinoma (HCC) is the major histological subtype among types of primary liver cancer, and

Correspondence to: Professor Jianguo $\mathrm{Lu}$ or $\mathrm{Dr}$ Xilin Du, Department of General Surgery, Tangdu Hospital, Fourth Military Medical University, 569 Xinsi Road, Xi'an, Shaanxi 710038, P.R. China

E-mail: lujguo@hotmail.com

E-mail: dxlin0705@163.com

*Contributed equally

Key words: hepatocellular carcinoma, mutations, next generation sequencing it is estimated that $>600,000$ cases of $\mathrm{HCC}$ are newly diagnosed each year worldwide, $>50 \%$ of which are in China $(1,2)$. Despite advances in diagnosis and multimodality therapies for HCC, the prognosis of the disease remains poor, with a 5 -year overall survival rate of $<14 \%$ (3).

The environmental risk factors for HCC include hepatitis B virus (HBV) and hepatitis C virus (HCV) infection, food aflatoxin contaminants, alcohol intake and smoking (2). $\mathrm{HBV}$ infection is the dominant global attributable risk factor, accounting for $>80 \%$ of the cases of HCC in China (2). HBV is small enveloped DNA virus, belonging to the hepadnavirus family, which is capable of integrating into and altering the host genome. A total of four overlapping open reading frames, encoding for the surface protein, core protein, polymerase protein and $\mathrm{X}$ protein (HBX), have been identified in the small $3.2 \mathrm{~kb}$ DNA of HBV $(4,5)$. HBX is the regulatory protein of the virus, and is frequently detected in the tumor tissues of patients with HBV-associated HCC $(4,5)$. HBX transgenic mice have been reported to exhibit a positive correlation between the expression levels of HBX and the development of HCC $(6,7)$. Consuming food containing aflatoxin is another high environmental risk factor for HCC. Aflatoxin B1 is the most abundant form of aflatoxin, produced by the fungus Aspergillus flavus, which contaminates food, particularly under hot and humid conditions (8-10). The metabolites of Aflatoxin B1 can form a DNA adduct at the third base of codon 249 in the TP53 gene, inducing a G>T transversion and an R249S mutation, which is the most frequently altered type of TP53 in HCC tissues (8-10).

Alterations in multiple signaling pathways and patterns of host gene expression have been documented following HBV infections. Somatic mutations, which trigger oncogenes or inactivate tumor suppressor genes, contribute to the development of HCC. Previous studies using several strategies have demonstrated that certain genetic alterations occur in HCC cells, including TP53, CTNNB1, Axin1 and ARID1A high frequency mutation genes and Kras and APC low frequency mutation genes (11-13). The development of next generation sequencing (NGS) has led to an increase in the number of mutations identified in HCC tissues. Huang et al (14) investigated the spectrum of molecular aberrations in liver cancer using whole exome sequencing, and identified 356 candidate somatic single nucleotide variants (SNVs) within 347 genes. 
Despite efforts to improve the diagnosis and treatment of HCC, the molecular mechanisms leading to the development and progression of $\mathrm{HBV}$-associated $\mathrm{HCC}$ remain to be fully elucidated. In addition, investigations focus predominantly on HCC samples from southern and eastern China, while molecular data from HCC samples in western China remains limited. Due to the different environmental factors, patients with HCC in western China may exhibit characteristic patterns of gene alterations.

In the present study, targeted sequencing of 372 genes was performed using an NGS platform, in order to investigate the mutation spectrum of HCC samples from western China. The investigation aimed to provide resources for understanding the molecular alterations associated with the development of HCC.

\section{Materials and methods}

Tissue samples. A total of 12 patients from western China, who had undergone surgery for HCC at Tangdu Hospital, Fourth Military Medical University (Xi'an, China) between December 2010 and September 2012, were included in the present study. The patients' cancerous and adjacent non-cancerous tissues (minimum, $3 \mathrm{~cm}$ ) were collected and analyzed in the present study. Both cancerous and non-cancerous samples were flash frozen in liquid nitrogen following surgery and then stored at $-80^{\circ} \mathrm{C}$, until DNA extraction. The size of tissue samples used for DNA extraction was $\sim 0.2 \times 0.2 \times 0.2 \mathrm{~cm}$. The majority of these patients were chronic HBV carriers. The study population consisted of 11 males and 1 female, with a median age of 51 years old (range 33-65 years). All the HCC cases were histopathologically confirmed. Cirrhosis and capsules were confirmed by histochemical analysis using a light microscope (CDM965; Shanghai Tuming Optical Instrument Co., Ltd., Shanghai, China). Information regarding family history, smoking history, and drinking history was acquired by questionnaire.

The experiments were performed with the understanding and written consent of each patient, and the investigation was performed in accordance with The Code of Ethics of the World Medical Association (Declaration of Helsinki), printed in the British Medical Journal (1964) (15). The present study was also approved by the Ethics Committee of Tangdu Hospital, Fourth Military Medical University.

Target enrichment of genomic DNA and sequencing. Genomic DNA was extracted using a QIAamp DNA Mini kit (cat. no. 51306), according to the manufacturer's instructions (Qiagen, Dusseldorf, Germany). The targeted regions included all exons of 372 genes. The targeted genes were selected from the Catalogue of Somatic Mutations in Cancer (Cosmic; http://www.sanger.ac.uk/genetics/CGP/cosmic) database using the 2011 updated edition. Illumina TruSeq technology was used to capture the exon region of 372 genes, using a TruSeq Custom Enrichment Trial kit, according to the manufacturer's instructions (Illumina, Inc., San Diego, CA, USA). An Illumina TruSeq DNA Sample Prep kit was used for TruSeq sample preparation, and the samples were then sequenced using the Illumina HiSeq platform at Shanghai ZhangJiang Translational Medicine Research Center (Shanghai, China).

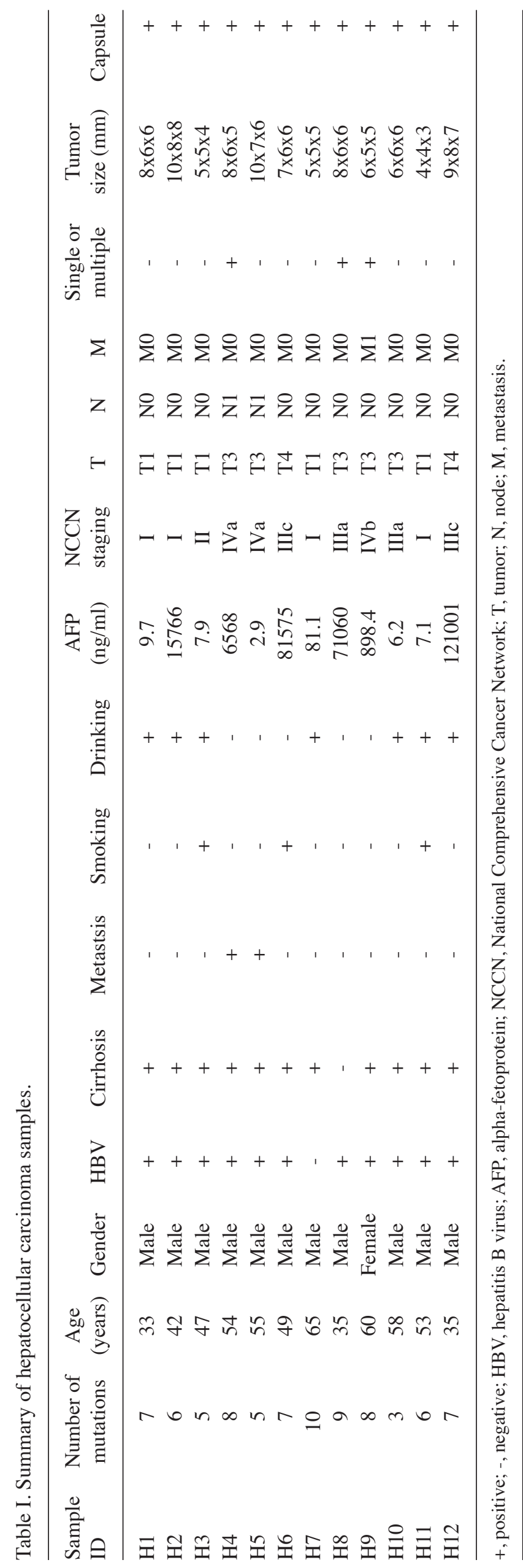


Data analysis. For each patient, DNA was extracted from both tumor tissues and non tumor tissues, and was sequenced at the same time. The SNVs and insertions/deletions were identified using a Genome Analyzer Toolkit (GATK, version 3.0; Broad Institute, Cambridge, MA, USA). Coverage represents the number of times that each nucleotide is sequenced at the NGS platform. Effective rate represents the ratio of clean data (following removal of low quality and contaminated data) to raw data. To identify somatic mutations, the sequence variants of the non tumor tissues were subtracted from those of the tumor tissues. The Database for Annotation, Visualization and Integrated Discovery (DAVID; http://david.abcc.ncifcrf.gov/) was used for pathway analysis.

\section{Results}

Sample characteristics. The study population comprised of 11 males and 1 female. Of these, 11 patients were chronic HBV carriers and 1 patient was not an HBV carrier. A total of 11 patients had liver cirrhosis and 1 patient had no cirrhosis, three patients had a history of smoking and seven patients had a history of drinking. All the HCC tumors were accompanied by tumor capsules, and tumor cell invasion was observed by microscopy. The patient characteristics are described in Table I.

Sequencing profiles. The genomic DNA obtained from 12 patients with HCC was screened for somatic mutations in 372 genes. On average, $0.197 \mathrm{~Gb}$ sequencing data was obtained for each sample. The average coverage of each base in the target regions was $\sim 40 \mathrm{x}$ per tumor sample, and the effective rate for each sample was $>86 \%$.

Mutation profiles. In total, 81 non-synonymous somatic mutations were identified in 62 different genes. Of the somatic mutations, 80 were heterozygous and 1 was homozygous. A total of 77 SNVs were observed in 58 genes from 12 patients and four deletion variations, including one frameshift mutation and three non-frameshift mutations, were identified in four genes from three patients. Point mutations identified included 67 missense mutations and 10 nonsense mutations. The HCC mutation spectrum was dominated by $\mathrm{C}>\mathrm{T}$ and $\mathrm{C}>\mathrm{A}$ transitions, and the median number of mutations in each tumor sample was seven (range, 3-10). Due to the small sample number, no significant association was observed between the mutation spectrum and clinical features. Deletion was observed in MAML2, NN1, RET and MLLT3 genes, and the deletion of MAML2 resulted in a frameshift alteration. No insertion mutations were observed in the present study.

Recurrent mutations. The two most frequently mutated genes in the present study were TP53 and RUNX1, with frequencies of 5/12 and 3/12, respectively. The mutations of TP53 were missense or nonsense and located in exons 2, 3 and 4, and four mutations were present in the transactivation domain (Fig. 1A). The mutations of RUNX1 were all missense, located in exons 1, 3 and 6, one of which was in the inhibitory domain (Fig. 1B). Additionally, 9 other genes, which were recurrently mutated in at least 2/12 tumors were identified (Table II). The $\mathrm{H} 7$ tumor exhibited two mutations in the FLCN gene, resulting
Table II. Recurrent mutations in hepatocellular carcinoma samples.

\begin{tabular}{|c|c|c|c|}
\hline Gene & $\begin{array}{l}\text { Mutation } \\
\text { frequency }\end{array}$ & Mutation & Sample \\
\hline TP53 & $5 / 12$ & $\begin{array}{c}\text { c.G422A:p.R141H, } \\
\text { c.T368C:p.I123T, } \\
\text { c.A229T:p.R77X, } \\
\text { c.G315A:p.M105I, } \\
\text { c.G351T:p.R117S }\end{array}$ & $\begin{array}{l}\mathrm{H} 12 \\
\mathrm{H} 8 \\
\mathrm{H} 9 \\
\mathrm{H} 4 \\
\mathrm{H} 5\end{array}$ \\
\hline RUNX1 & $3 / 12$ & $\begin{array}{c}\text { c.T1277A:p.V426E, } \\
\text { c.C167T:p.A56V, } \\
\text { c.A64G:p.I22V }\end{array}$ & $\begin{array}{l}\mathrm{H} 3 \\
\mathrm{H} 7 \\
\mathrm{H} 4\end{array}$ \\
\hline JAK & $2 / 12$ & $\begin{array}{l}\text { c.C3124T:p.R1042W, } \\
\text { c.G3205T:p.E1069X }\end{array}$ & $\begin{array}{l}\mathrm{H} 1 \\
\mathrm{H} 3\end{array}$ \\
\hline CREBBP & $2 / 12$ & $\begin{array}{l}\text { c.G2103C:p.M701I, } \\
\text { c.G6291T:p.Q2097H }\end{array}$ & $\begin{array}{l}\mathrm{H} 7 \\
\mathrm{H} 9\end{array}$ \\
\hline FLCN & $2 / 12$ & $\begin{array}{c}\text { c.G1536T:p.M512I, } \\
\text { c.G268T:p.A90S }\end{array}$ & $\mathrm{H} 7$ \\
\hline HOXC13 & $2 / 12$ & $\begin{array}{l}\text { c.C71A:p.A24E, } \\
\text { c.A425T:p.Q142L }\end{array}$ & $\begin{array}{l}\mathrm{H} 6 \\
\mathrm{H} 5\end{array}$ \\
\hline KRAS & $2 / 12$ & $\begin{array}{c}\text { c.G507T:p.K169N, } \\
\text { c.G38A:p.G13D }\end{array}$ & $\begin{array}{l}\mathrm{H} 5 \\
\mathrm{H} 2\end{array}$ \\
\hline MECOM & $2 / 12$ & $\begin{array}{l}\text { c.A197T:p.N66I, } \\
\text { c.A725G:p.K242R }\end{array}$ & $\begin{array}{l}\mathrm{H} 7 \\
\mathrm{H} 9\end{array}$ \\
\hline NF1 & $2 / 12$ & $\begin{array}{c}\text { c.A7382T:p.D2461V, } \\
\text { c.G1716T:p.E572D }\end{array}$ & $\begin{array}{c}\mathrm{H} 12 \\
\mathrm{H} 6\end{array}$ \\
\hline NOTCH1 & $2 / 12$ & $\begin{array}{c}\text { c.G236A:p.R79H, } \\
\text { c.C7397T:p.T2466M }\end{array}$ & $\begin{array}{l}\mathrm{H} 8 \\
\mathrm{H} 1\end{array}$ \\
\hline PDE4DIP & $2 / 12$ & $\begin{array}{c}\text { c.G4111A:p.V1371I, } \\
\text { c.G512A:p.R171K }\end{array}$ & $\begin{array}{c}\mathrm{H} 3 \\
\mathrm{H} 11\end{array}$ \\
\hline USP6 & $2 / 12$ & $\begin{array}{c}\text { c.G1573A:p.V525I, } \\
\text { c.C215G:p.T72R }\end{array}$ & $\begin{array}{l}\text { H9 } \\
\text { H11 }\end{array}$ \\
\hline RECQL4 & $2 / 12$ & $\begin{array}{l}\text { c.T274C:p.S92P, } \\
\text { c.G187T:p.E63X }\end{array}$ & $\begin{array}{l}\mathrm{H} 8 \\
\mathrm{H} 8\end{array}$ \\
\hline
\end{tabular}

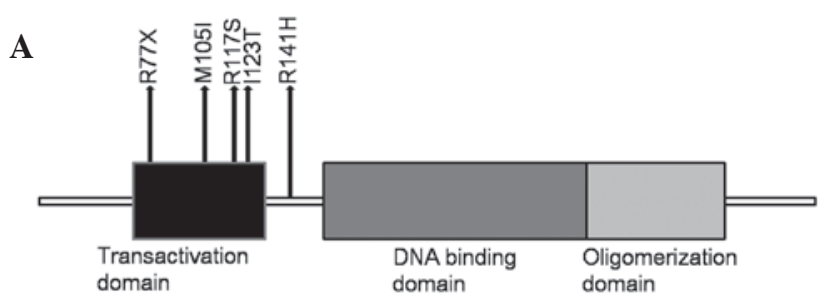

B

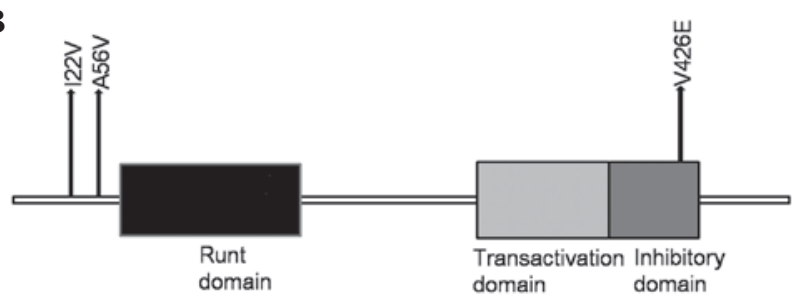

Figure 1. TP53 and RUNX1 somatic mutations in hepatocellular carcinoma samples. (A) Status of somatic mutations observed in TP53. (B) Status of somatic mutations observed in RUNX1. Arrows indicate mutation positions. 
in c.G268T:p.A90S and c.G1536T:p.M512I, while H8 had two mutations in the RECQL4 genes, resulting in c.T274C:p.S92P and c.G187T:p.E63X.

Pathways. Pathway analysis was performed for genes containing mutations in each tumor using the DAVID database. A total of 77 altered genes were analyzed. Following the exclusion of disease-associated pathways, including pathways in cancer, six pathways were found to be significantly involved: The Janus kinase (JAK)-signal transducer and activator of transcription (STAT) signaling pathway (hsa04630), neurotrophin pathway (hsa04722), apoptosis pathway (hsa04210), focal adhesion signaling pathway (hsa04510), notch signaling pathway (hsa04330) and p53 signaling pathway (hsa04115). Among these pathways, the JAK-STAT signaling pathway was the most frequently involved, with six patients (50\%) exhibiting possible altered function in this pathway.

\section{Discussion}

Multiple genetic events accumulate during the progression of HCC development. In the present study, the profile of genetic alterations in HCC was analyzed using a NGS platform. In total, 372 genes from 12 pairs of normal and tumor tissue specimens were sequenced and a total of 81 non-silent somatic point mutations were found.

The samples used in the present study were from patients from western China, therefore, the profiling of the mutation spectrum performed was, to a certain extent, different from previous studies $(8-10,13,14)$. The two most commonly mutated genes observed in the present study were TP53 and RUNX1. The TP53 gene, termed 'the guardian of the genome' has been reported to be the most frequently altered gene in various types of cancer, including HCC (16). In the present study, the mutation frequency of TP53 was $\sim 41.7 \%$. However, the distribution of mutations was different from that observed in previous studies $(8-10,13,14)$. The $\mathrm{p} 53$ protein can be divided into three main domains, the transactivation domain (exons 2 and 3), the DNA binding domain (exons 5-8) and the oligomerization domain (exons 9 and 10). The transactivation domain, at the $\mathrm{N}$ terminus of the protein, is rich in serine and threonine and is able to induce protein activation. The DNA binding domain recognizes and binds a consensus sequence in the promoter sequence of genes, which is regulated by p53 at the transcriptional level $(17,18)$. The oligomerization domain, at the $\mathrm{C}$ terminus of the $\mathrm{p} 53$ protein, assists in the formation of an active tetramer $(17,18)$. Previous studies (8-10) have reported that the majority of p53 mutations are localized in the DNA binding domain, and that R249 was the most common site for this, which has been observed in $>30 \%$ of HCC cases in geographical areas of high HCC incidence. The R249S mutation is associated with the induction of AFB1 (8-10). AFB1 is the metabolite of aflatoxin-producing fungi, which is able to form a DNA adduct at the third base of codon 249 in the TP53 gene, inducing a G>T transversion and an R249S mutation $(8,9)$. In an area with a hot and humid climate, aflatoxin-producing fungi are able to grow and can contaminate food, including corn and peanuts, therefore the AFB1-inducing mutation R249S is commonly observed (8-10). In the present study, all the mutations identified were in exons 2, 3 and 4, which is possibly due to the fact that the samples were from patients from western China, facing different environmental factors and, thus, exhibiting a different mutation spectrum for p53. RUNX1 is located on chromosome 21q22 and contains an 138 amino acid Runt homology domain, which is necessary for DNA consensus sequence recognition and interaction with other co-factors (19). RUNX1 is able to increase or inhibit transcriptional activity of target genes. Its roles include regulating cell differentiation, growth and survival (20). A previous study (21) reported that RUNX1 was one of the most common targets of chromosomal rearrangement in leukemia, and was dysregulated in certain types of solid tumor. Miyagawa et al (22) reported that RUNX1 was downregulated in HCC tissue, however, to the best of our knowledge no mutation of RUNX1 in HCC has been previously reported. In the present study, three RUNX1 mutations were identified, suggesting its importance in liver carcinogenesis. In addition, the present study identified mutations in several cancer genes, which had not been previously linked to HCC, including JAK3. JAK3 is a member of the non-receptor tyrosine kinase family, the members of which are able to bind to various cell surface receptors and are important in cytokine-induced signal transduction $(23,24)$. Previous studies $(24,25)$ have reported that JAK3, unlike the JAK1, JAK2 or Tyk2 members of the non-receptor tyrosine kinase family, is primarily expressed in cells of a hematopoietic lineage, and mutations of JAK3 have been identified in leukemic patients and cell lines. In the present study, two non-synonymous mutations for JAK3 were identified in patients with HCC. This, to the best of our knowledge, is the first time that mutations of JAK3 have been reported in HCC, suggesting a novel role of JAK3.

In conclusion, the present study identified several novel genes involved in HCC using NGS. The results of the present study provide a resources for understanding the molecular alterations underlying the development of HCC, however, further investigations, with larger sample sizes, are required to fully examine genetic alteration in HCC development.

\section{Acknowledgements}

The authors would like to thank Shanghai ZhangJiang Translational Medicine Research Center for their assistance with data analysis.

\section{References}

1. Siegel R, Ward E, Brawley O and Jemal A: Cancer statistics, 2011: The impact of eliminating socioeconomic and racial disparities on premature cancer deaths. CA Cancer J Clin 61: 212-236, 2011.

2. Llovet JM, Burroughs A and Bruix J: Hepatocellular carcinoma. Lancet 362: 1907-1917, 2003.

3. Center MM and Jemal A: International trends in liver cancer incidence rates. Cancer Epidemiol Biomarkers Prev 20: 2362-2368, 2011.

4. Kim CM, Koike K, Saito I, Miyamura T and Jay G: HBx gene of hepatitis B virus induces liver cancer in transgenic mice. Nature 351: 317-320, 1991.

5. Koike K, Moriya K, Iino S, et al: High-level expression of hepatitis $\mathrm{B}$ virus HBx gene and hepatocarcinogenesis in transgenic mice. Hepatology 19: 810-819, 1994.

6. Bouchard MJ and Schneider RJ: The enigmatic X gene of hepatitis B virus. J Virol 78: 12725-12734, 2004. 
7. Seeger C and Mason WS: Hepatitis B virus biology. Microbiol Mol Biol Rev 64: 51-68, 2000.

8. Gouas D, Shi H and Hainaut P: The aflatoxin-induced TP53 mutation at codon 249 (R249S): Biomarker of exposure, early detection and target for therapy. Cancer Lett 286: 29-37, 2009.

9. Hsu IC, Metcalf RA, Sun T, Welsh JA, Wang NJ and Harris CC: Mutational hotspot in the p53 gene in human hepatocellular carcinomas. Nature 350: 427-428, 1991.

10. Shen HM and Ong CN: Mutations of the p53 tumor suppressor gene and ras oncogenes in aflatoxin hepatocarcinogenesis. Mutat Res 366: 23-44, 1996.

11. Li M, Zhao H, Zhang X, et al: Inactivating mutations of the chromatin remodeling gene ARID2 in hepatocellular carcinoma. Nat Genet 43: 828-829, 2011.

12. Boyault S, Rickman DS, de Reyniès A, et al: Transcriptome classification of HCC is related to gene alterations and to new therapeutic targets. Hepatology 45: 42-52, 2007.

13. Zender L, Villanueva A, Tovar V, Sia D, Chiang DY and Llovet JM: Cancer gene discovery in hepatocellular carcinoma. J Hepatol 52: 921-929, 2010.

14. Huang J, Deng Q, Wang Q, et al: Exome sequencing of hepatitis $B$ virus-associated hepatocellular carcinoma. Nat Genet 44: $1117-1121,2012$

15. Gandevia B and Tovell A: Declaration of Helsinki. Med J Aust 2: $320-321,1964$

16. Levrero M, De Laurenzi V, Costanzo A, Gong J, Wang IY and Melino G: The p53/p63/p73 family of transcription factors: Overlapping and distinct functions. J Cell Sci 113: 1661-1670, 2000 .
17. Laptenko $\mathrm{O}$ and Prives C: Transcriptional regulation by p53: One protein, many possibilities. Cell Death Differ 13: 951-961, 2006.

18. Cho Y, Gorina S, Jeffrey PD and Pavletich NP: Crystal structure of a p53 tumor suppressor-DNA complex: Understanding tumorigenic mutations. Science 265: 346-355, 1994.

19. Rossetti S and Sacchi N: RUNX1: A MicroRNA hub in normal and malignant hematopoiesis. Int J Mol Sci 14: 1566-1588, 2013.

20. Blyth K, Cameron ER and Neil JC: The RUNX genes: Gain or loss of function in cancer. Nat Rev Cancer 5: 376-387, 2005.

21. Meyers S, Downing JR and Hiebert SW: Identification of AML-1 and the $(8 ; 21)$ translocation protein (AML-1/ETO) as sequence-specific DNA-binding proteins: The runt homology domain is required for DNA binding and protein-protein interactions. Mol Cell Biol 13: 6336-6345, 1993.

22. Miyagawa K, Sakakura C, Nakashima S, et al: Down-regulation of RUNX1, RUNX3 and CBFbeta in hepatocellular carcinomas in an early stage of hepatocarcinogenesis. Anticancer Res 26: 3633-3643, 2006

23. Yamaoka K, Saharinen P, Pesu M, Holt VE III, Silvennoinen O and O'Shea JJ: The Janus kinases (Jaks). Genome Biol 5: 253, 2004.

24. Pesu M, Laurence A, Kishore N, Zwillich SH, Chan G and O'Shea JJ: Therapeutic targeting of Janus kinases. Immunol Rev 223: 132-142, 2008.

25. Elliott NE, Cleveland SM, Grann V, Janik J, Waldmann TA and Davé UP: FERM domain mutations induce gain of function in JAK3 in adult T-cell leukemia/lymphoma. Blood 118: 3911-3921, 2011. 